\title{
3D interactive environment for the design of medical devices
}

\author{
Giorgio Colombo ${ }^{1} \cdot$ Caterina Rizzi ${ }^{2} \cdot$ Daniele Regazzoni $^{2} \cdot$ Andrea Vitali $^{2}$
}

Received: 3 November 2017 / Accepted: 12 January 2018 / Published online: 29 January 2018

\begin{abstract}
The effectiveness of custom-made prostheses or orthoses heavily depends on the experience and skills of the personnel involved in their production. For complex devices, such as lower limb prosthesis, a conventional manual approach affects the process at the point that the result is frequently not acceptable at the first trial. The paper presents a computer-aided environment, named socket modelling assistant ${ }^{2}$ (i.e., $\mathrm{SMA}^{2}$ ), to interactively design the socket of lower limb prosthesis by implementing a set of design rules extrapolated from the traditional development process. The new computer-aided environment has been implemented embracing a low-cost philosophy and using open source libraries to provide a solution affordable also by small orthopaedic laboratories. The system permits to modify and interact with the 3D model of residual limb to create the socket geometric model ready to be manufactured by means of additive manufacturing. SMA ${ }^{2}$ embeds medical knowledge related to the device functioning, the conventional process and the way orthopaedic technicians work so that it can be much more reliable and repeatable compared to the conventional process, but still enough similar to it to be accepted by the involved personnel. In the paper, the new 3D design procedure is described in detail, from the acquisition of patient's data to preliminary and customized modelling, and new geometric tools to perform context-related operations are shown. A case study is used to clarify the way the system works and to provide an example of the outcome.
\end{abstract}

Keywords Interactive design · Geometric modelling · Custom medical devices · Additive manufacturing

\section{Introduction}

In the last 25 years the introduction of CAD/CAM systems in orthotic and prosthetic (O\&P) industry allowed improving medical devices quality and thus the quality of life of patients with severe conditions such as limb amputation. Typically, CAD/CAM systems for O\&P industry, include three main components: an acquisition system, a software application for prosthesis design and a carving or milling machine. The first system allows 3D acquiring of the anatomical district; the CAD application permits to design the orthopaedic device by

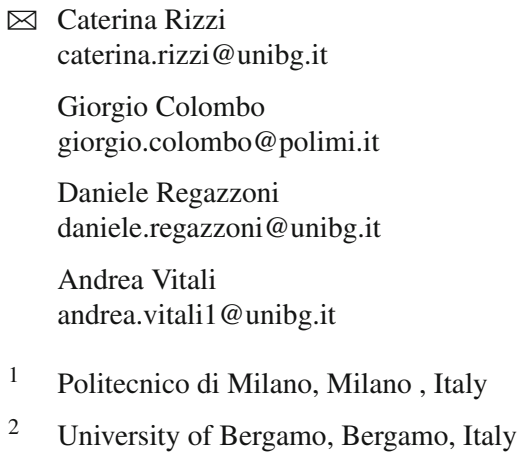

starting from the 3D virtual model of the human body district. When the 3D model of designed device is ready, it is used for creating the O\&P device by using either a carving or milling machine.

From one hand, this approach may improve the quality of O\&P devices and accelerate the production but, on the other hand, these systems are expensive and not easily accessible by O\&P laboratory with limited economic resource. Furthermore, not all the orthopaedic technicians have the adequate background on ICT technologies to properly use CAD/CAM systems, especially in small orthopaedic labs, which are common in Italy. In this context, the orthopaedic technicians acquire high professional skills in prosthesis manufacturing after many years of experience and the whole product development process is, usually, based on a hand-made approach. For example, during the design of lower limb prosthesis the technicians empirically use a set of rules and knowledge, which are not embedded within the existent commercial CAD/CAM systems. Even if most of the components of the lower limb prosthesis are standard, the most important component, i.e., the socket, is heavily customized and depends 
on patients history, which is, by definition, different from person to person.

The socket design depends on several aspects related to the morphology of the residual limb and patient's life style, which are faced and solved by the technicians thanks to their experience and know-how. However, commercial O\&P CAD/CAM systems embed few design rules for lower limb prosthesis and still rely on technician's know-how.

This paper presents a new software platform, named socket modelling assistant ${ }^{2}$ (i.e., $\mathrm{SMA}^{2}$ ) finalised to improve the interactive creation and manipulation of the socket shape around the digital model of the residual limb making possible a high level of product customisation and ensuring the prothesis functionality and comfort. $\mathrm{SMA}^{2}$ is part of an interactive design platform centred on the virtual model of the amputee and based on a computer-aided and knowledgeguided approach. It is the kernel of the whole system and embeds design rules and knowledge acquired from the prosthetists. It comprehends several modelling tools, which emulate the traditional manufacturing operations executed by the technicians. Its main goal is to provide an interactive environment usable by people without specific skills on 3D modelling/simulation tools and mathematical models.

This is possible due to the use of innovative low-cost devices as well as open source development kits which may be used to develop new interaction styles very near to the real ones. $\mathrm{SMA}^{2}$ development has been based on a previous version that has been tested in collaboration with prosthetists and engineering students [11]. This permitted us to verify the potential of our approach and in the meantime to identify new functionalities and software development toolkits to implement them.

$\mathrm{SMA}^{2}$ can be considered an application of Interactive Design and Manufacturing paradigm since it is focused on tools which allows the users to create and interactively modify the geometry of both the residual limb (reference model) and the socket in real time.

The paper presents a state of the art review on 3D acquisition and modelling, on 3D simulation and on additive manufacturing, all related to the development of innovative devices for medical use and for prostheses and orthoses. After the state of the art, the traditional process for creating a lower limb prosthesis is shown. Such a process is analysed to define the new software architecture, the new interactive modelling tools and the way simulations are performed. Once all specific tools have been described, the new proposed procedure is shown from the beginning to the result, including the manufacturing with additive multi-material technology. The last part of the paper refers to the application to a real case study with a transfemoral patient and then conclusions are drawn.

\section{Scientific background}

The proposed solution covers all steps of the socket development process from residuum acquisition to its manufacturing by using Additive Manufacturing technologies. A low-cost philosophy has been considered for each step-in order to offer a solution affordable also by small orthopaedic labs and, at same time, innovative and of high quality. In this section, we first introduce the existing commercial solutions and then we discuss the state of art of main scientific issues considered to develop SMA ${ }^{2}$. Four main issues have been considered: residual limb acquisition, socket modelling, socket-residuum interaction and additive manufacturing.

\subsection{D acquisition}

The reverse engineering is the technique with which we can obtain a 3D virtual model of an object by using either a 3D scanner [26] or medical imaging devices (e.g. magnetic resonance imaging, MRI) [3,43].

There are many types of 3D scanners that can be classified according to several features, such as resolution, accuracy, portability and cost [4]. The 3D reconstruction by medical imaging depends on the technology used for acquiring the residual limb of the patient. Both 3D scanners and MRI present pros and cons relative to adopted technology. 3D scanner permits to acquire in very accurate way the undeformed geometry of the residual limb, but no information can be obtained relative to the internal parts of the residual limb. MRI images permit to get useful data relative internal tissues of the limb, but MRI images are usually obtained with patient lying down on a rigid bed and this causes the flattening of the thigh.

External 3D scanners can be divided in industrial solutions and low-cost solutions. There are several industrial 3D scanners used to acquire the shape of the residual lower limb. The most used are Vorum Spectra Scanner, Willowood 3D scanner and Rodin scanners, which guarantee a high quality of 3D model in terms of precise recognition of details.

On the other side, low cost solutions are available for the same aim. Microsoft Kinect v1 can be exploited as 3D scanner through the application Skanect. Furthermore, a structure sensor can be plugged to an Apple device to be used as a 3D scanner. Even if these solutions acquire with a lower quality than industrial solutions, the 3D mesh is good enough for the final purpose, which is the use of the 3D model of the residual limb as starting point to model the final shape of the socket.

Computed tomography (CT) is the most suitable technique from the 3D modelling point of view because any grey level is directly associated to a specific tissue and, thus, automatic reconstruction is possible. Indeed, there are commercial solutions (e.g. Mimics by Matirialize [29]) providing automatic 
reconstruction functionalities. However, CT is cannot be used frequently because it is based on X-rays and it is invasive for patients. MRI technology, instead, can be done several times without medical complications for patients, but the data obtained, in term of grey level, depends on the acquisition protocol and makes the reconstruction much more challenging. 3D reconstruction from MRI is a technique used in several medical sectors for improving diagnosis of medical staff. At present, there are some software systems aimed at creating some 3D model of the interested human districts. These applications are based on the use of complex image processing algorithms, which allow extracting profiles relative to a particular object (e.g., a bone, muscle tissues and tendons) by each 2D image of the MRI volume. Complex procedures are employed to detect and merge profiles to create a $3 \mathrm{D}$ point cloud used to create the mesh model.

Some commercial solutions are Osirix [40], Materialise Mimics, Amira Avizo 3D software [48] and Radiant Dicom [38]. Moreover, also some open source applications have been developed for 3D reconstruction, such as 3D Slicer [1], MITK [30], DeVide [35], Invesalius 3 [25]. Among them, the most important tools are Osirix and 3D Slicer that can be used for diagnosis $[14,16,22,44]$, surgery $[7,16,32,41]$ and $3 \mathrm{D}$ reconstruction of organs for $3 \mathrm{D}$ printing and medical evaluation [18,31].

By the way, there are no commercial tools providing an automatic, one-click procedure of $3 \mathrm{D}$ reconstruction from MRI suitable for our aim.

\subsection{D modelling}

Some of the most important software houses involved in medical devices design are Vorum, Rodin4D, Ohio Willow Wood and Nia Technologies.

Vorum [49] offers a complete suite composed by an industrial 3D scanner, CAD application and CAM system in order to design O\&P devices among which the socket for lower limb prosthesis. In particular, Vorum has introduced the use of additive manufacturing for creating sockets. Also, Rodin4D [39] offers complete CAD/CAM systems and is doing research to develop innovative technologies for socket design.

Ohio Willow Wood offers the use of low cost scanner in addition to classic CAD/CAM solution. The low-cost 3D scanner Structure Sensor permits the 3D acquisition of object using smartphones and tablets [52].

Another important reality is Nia Technologies Inc., a Canadian not-for-profit organization specialized in the creation of prosthetic sockets and orthotic braces for children [33]. Basically, they exploit a custom version of AutoDesk MeshMixer [2,17] as 3D CAD system and Microsoft Kinect v1 as 3D scanner.
The approach proposed in this paper differs from the existing ones mainly because it is centred on the virtual patient seen from a medical perspective and it aims at providing new tools derived from a complex engineering approach but with a sharp focus on usability $[6,19,36]$. To reach this aim, we did not start by modifying existing tools, but we deeply analyse the conventional working procedures to build a consistent alternative process based on geometric modelling and simulation. Thus, a virtual environment has been created embedding physicians and technicians' knowledge and best practices into a new design paradigm where tools have been created coherently.

In the next section, our approach is described with emphasis on knowledge-guided process and low-cost philosophy.

\subsection{Simulation}

Many research works have shown attempts of introducing FEA into the prosthesis simulation since 2000. Simulations are mainly aimed at determining the level of performance in terms of functionality and comfort of the socket during donning, standing and walking activities. Thus, the interaction between the residual limb and the socket are investigated, mainly concerning forces and contact pressures. In most of literature contributions, researchers manually create linear or non-linear geometric models and simulate load conditions through commercial applications as Abaqus and ANSYS. By the way, a standalone simulation system has a limited impact on the design process since it is not trivial to connect simulation outcomes to required changes to improve the socket. This is the reason why we decided to create an integrated environment that embeds knowledge of the medical staff about physiology of the residual limb. It is crucial to know where load can be put, and which parts of the limb cannot be pressed because they could create blood circulation issues or pain.

Moreover, the introduced novelty consists also in having a simulation system which is able after results are generated to interpret them, through a set of rules, and to pass instructions to the modeller in order to fix problems by providing a better shape of the contact surface.

Thus, simulation and modelling can be iterated automatically until the desired level of performance and comfort are reached. There are no other known solution providing the same level of automation based on medical knowledge in a design environment.

\subsection{Additive manufacturing}

Additive manufacturing (AM) technologies are transforming traditional processes of designing and manufacturing products $[5,15,20,27,51,53]$. A wide range of solution is available on the market relative to $3 \mathrm{D}$ printing solutions and materials. 3D printing can be used in many contexts, for home, 
industrial and research applications. As a consequence to a large diffusion, many materials are available on the market. The most diffused low-cost technology for additive manufacturing is Fused Deposition Modelling (FDM), a printing technology that creates an object by extruding fused polymeric material.

At present, several research works have been proposed to introduce the use of additive manufacturing as an alternative way for socket production [13,23,42]. During last years, several techniques have been investigated to make additive technologies a viable solution. However, the main challenge and benefits are related to the chance of innovating product design through a Design for AM approach, for instance by vary the material mix in different parts of the product. Data driven AM exploits external data (e.g., forces, weight, pressures etc.) to optimize the structure of the printed model. Furthermore, this approach allows the evaluation of the mix of the materials to be used to obtain particular mechanical features of the 3D printed object (e.g., local mechanical properties).

The proposed solution allows printing a multi-material socket in which the choice of materials is guided by simulation results and anthropometric information of the patient.

\subsection{Proposed approach}

The proposed solution emulates traditional workflow usually done by orthopaedic technicians in laboratory. All rules relative to socket design have been embedded inside the virtual platform with the aim to transform many operations (e.g., 3D modelling operations, 3D reconstruction, FEA analysis) from manual to automatic or semi-automatic and very simple to be performed. The developed virtual platform is totally based on low-cost technology and open source SDKs. It allows the use of a Microsoft Kinect v1 as external laser scanner and includes an automatic procedure to create $3 \mathrm{D}$ models of external skin and bones from MRI images of the residual limb. A knowledge-guided CAD system has been developed that encapsulates socket design rules and makes available a set of virtual tools to automatically design the socket. A simulation module is automatically executed to evaluate the socket fitting procedure and, if necessary, to correct the socket shape according to obtained outcomes. Finally, the platform allows 3D printing of socket with single material or through data driven multi-material printing approach .

\section{Traditional manufacturing procedure}

There are two main types of lower limb prosthesis according to the level of the amputation: below knee (Fig. 1a) and above knee (Fig. 1a).
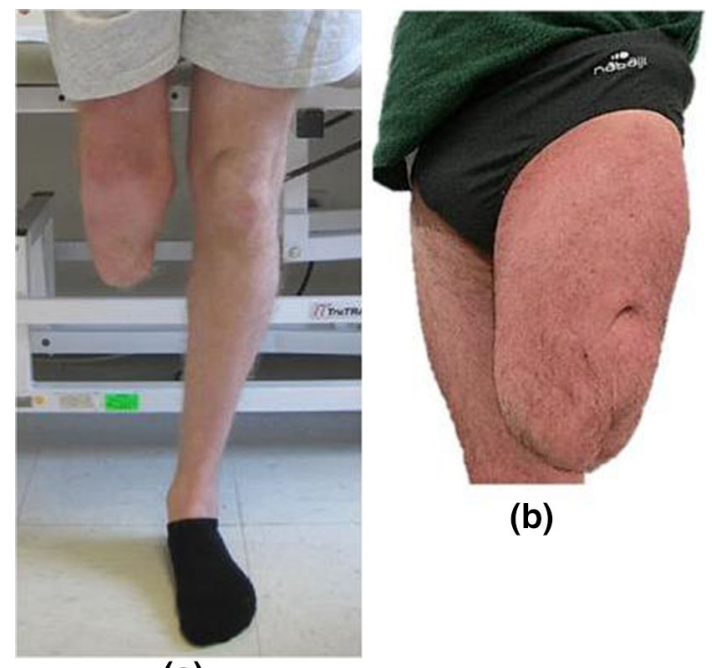

(b) (a)

Fig. 1 A below knee amputation (a) and an above knee amputation (b)

In previous research works $[8,10,11]$, from the analysis of traditional manufacturing process and interviews with prosthetists, we have acquired and formalized a set of rules and procedures to design lower limb prosthesis, in particular regarding the socket.

The traditional manufacturing starts from the evaluation of the amputee's residual limb and the creation of the negative cast by pressing with hands plaster patches directly on patient's residual limb. Then, he/she manufactures the positive plaster cast by adding and removing chalk in specific zones according to tacit knowledge and rules depending on residual limb morphology and patient's evaluation (e.g., lifestyle, residuum tonicity). In the virtual approach, the residual limb can be acquired by using MRI, CT and/or laser scanner (for details about the three solutions see [21,24,28]). In general, MRI is preferred since it is less invasive for the patient. The reconstructed 3D model represents in some way the positive cast around which the 3D model of the socket is created.

Three main operations can be identified:

- Initial plaster circumference reduction according to residual limb conditions (Fig. 2a). This means that the 3D residuum model has to be reduced. This operation should be done in automatic considering the residuum tonicity. For example, the socket must be more fitting for young or recently amputated patients, while for elderly patients it needs to be a bit loose to allow an easier gait or rehabilitation activities.

- Identification, marking and manipulation of critical zones (Fig. 2b ,c). The technician marks with a pencil the areas that must be modified and add or remove material in highlighted critical zones. Therefore, the system should 


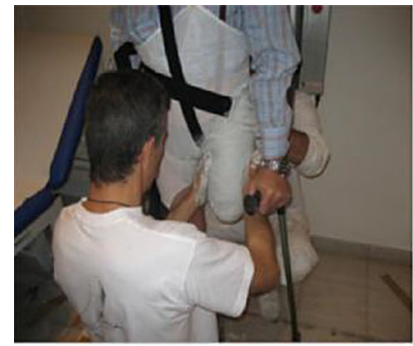

(a)

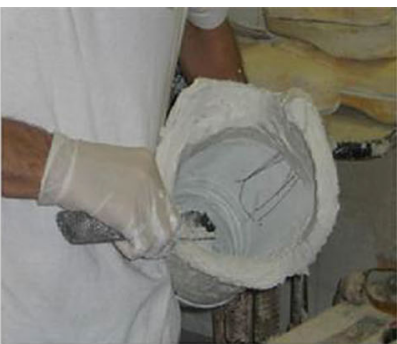

(b)

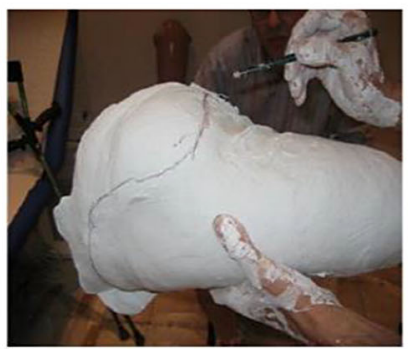

(c)

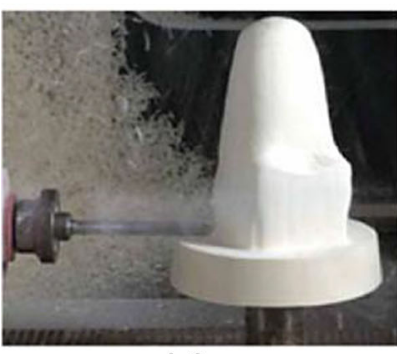

(d)

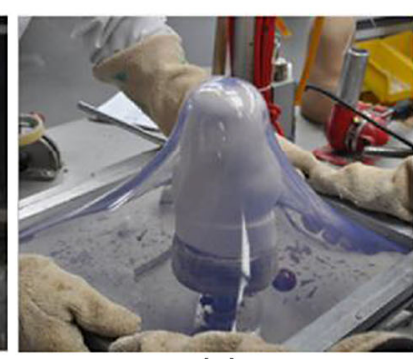

(e)

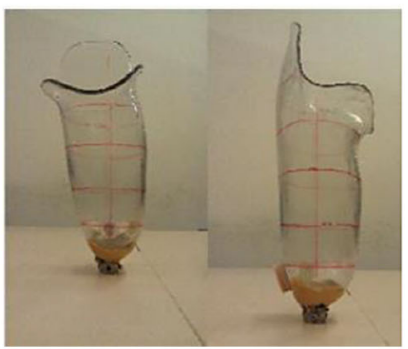

(f)

Fig. 2 a Plaster band positioning, b raw cast, $\mathbf{c}$ marking of critical zones, $\mathbf{d}$ creation of positive model, e laminating of check socket, $\mathbf{f}$ check socket ready

provide modelling tool that permits to emulate this operations on the virtual model of the residual limb considering also the possibility to use hands to model the shape.

- Then, the positive chalk model is created (Fig. 2d). The positive model has been exploited for the construction of a check socket to be tested with the patient (Fig. 2e, f). If required, minor changes are done to realize a more comfortable and well-fitting final socket. Finally, the definitive socket is laminated, and all the prosthesis components are assembled.
To reproduce the traditional manufacturing process some other rules have been defined connected to patient's data, such as lifestyle, anatomical situation of the residual lower limb (e.g., scars and muscle tonicity). Further rules have been considered in relation to innovative technology used for socket design using SMA.

\section{$4 \mathrm{SMA}^{2}$}

$\mathrm{SMA}^{2}$ is a knowledge-guided virtual platform that we developed for socket design. By starting from the 3D model of the residual limb, $\mathrm{SMA}^{2}$ makes available a set of automatic or semi-automatic virtual modelling tools to emulate traditional operations made by orthopaedic technicians. Furthermore, innovative technologies have been combined to assess the quality of the final 3D printed socket. Figure 3 shows the architecture of $\mathrm{SMA}^{2}$ interactive modelling platform.

Regarding software, $\mathrm{SMA}^{2}$ has been implemented in $\mathrm{C}++$ language using open-source SDKs such as:

- VTK [50], Visualization Tool Kit that allows modelling techniques, polygon reduction, cutting, mesh smoothing, contouring. This SDK also supports parallel computing for application exploiting high performance computing.

- Qt [37], a cross-platform application framework, which permits creating Graphical User Interface (GUI) in a very simple way. Qt is totally integrated by VTK.

- OpenCascade [34] has been mainly used for its exporting modules, which permit to save socket model in either STL or IGES format mainly used for contact pressure analysis.

Using object oriented paradigms, each virtual modelling tool is an autonomous software entity with which the other ones communicate by data relative to knowledge rules, patient's life style as well as previous modifications of the initial 3D model. This approach allows adding/removing new virtual tools according to feedback and needs of orthopaedic laboratories.

Regarding hardware, we have considered low cost solutions for both 3D scanning and additive manufacturing. Microsoft Kinect V1 and the commercial application Skanect have been considered to acquire and create the geometric model of the residual limb as an alternative to the 3D reconstruction by a MRI volume.

MRI has been considered since it is less invasive for the patient than Computed Tomography. A software module of SMA $^{2}$ automatically reconstruct mesh surfaces of both external skin and bones of the residual lower limb from MRI volume. Reconstructed 3D surfaces can be used for FE analysis of pressure behaviour between socket and residual limb during either donning phase or gait. The Leonardo 300 Cube" 
Fig. 3 Software architecture of SMA $^{2}$

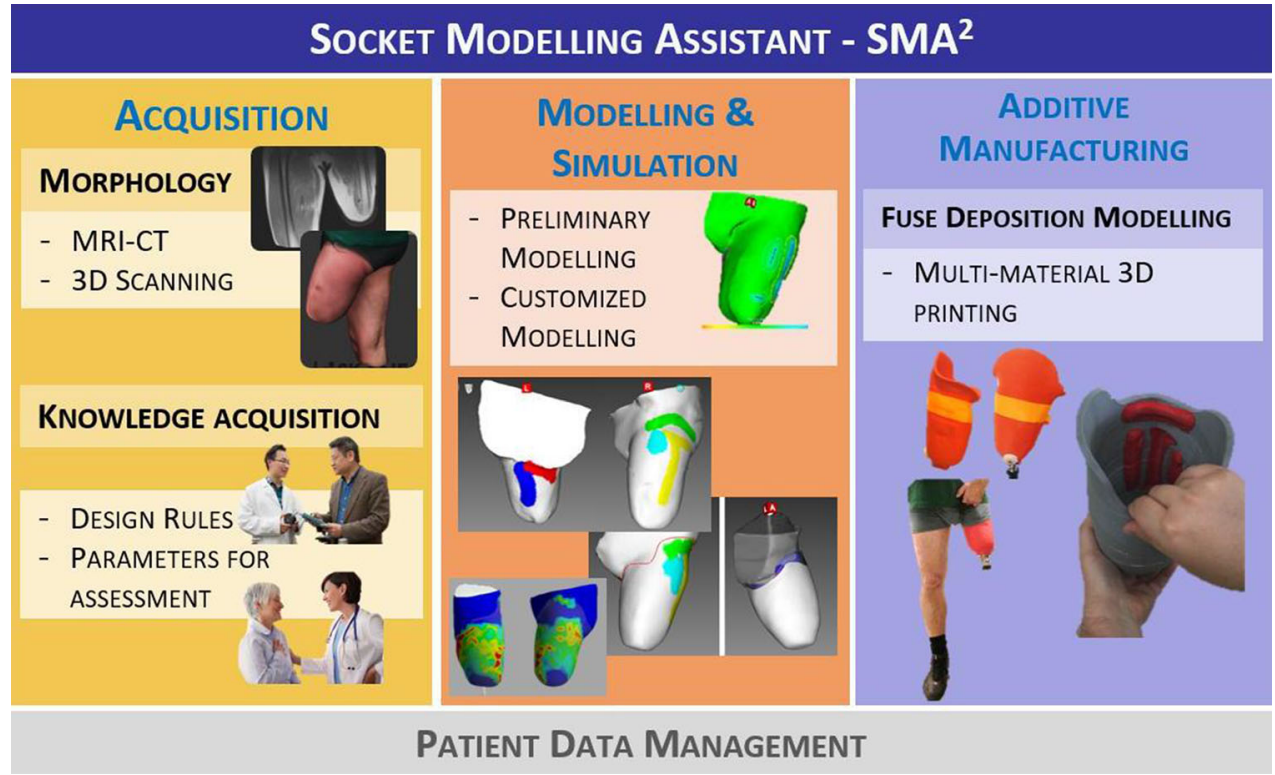

3D FDM printer by Meccatronicore has been used to create the socket physical prototype using the multi-material printing technology. Within $\mathrm{SMA}^{2}$ environment, the $3 \mathrm{D}$ geometric models of residual limb and socket are based on triangular meshes.

This approach required the software development of specific features to emulate real tools and operations carried out to create the socket. In the followings, the three main tools, which permit to emulate real operations and real tools used by orthopaedic technicians are described. They are: contour widget, sculpting tool and trim-line tool.

\subsection{Contour widget}

During the traditional manufacturing process, the orthopaedic technicians take measurements on the surface of the positive model to check if corrections are needed. Two types of measurements have been identified: the first one is the length of the circumferences on the transverse plane and the second one is the distance of two custom points along the surface of 3D model of the residual limb.

In the first case, we have exploited several functionalities made available by VTK, such as vtkPlaneSource, vtkPlane and vtkCutter. The first two classes permit to visualize the plane on which the transverse profile lies. vtkCutter class allows the computation of contour using the implicit function defined by vtkPlane class. vtkCutter allows to "slice-through" the surface of the 3D model, generating a contour that can be used to calculate the perimeter of the profile (Fig. 4).
Generated contour contains a set of 3D points ordered in the $3 \mathrm{D}$ space, which are used to calculate the perimeter by summing the length of each side composing the polygon. The custom contour widget has been also exploited for data driven multi-material 3D printing (see Sect. 5.3).

The virtual tape measure is an evolution of the vtkContourWidget. The contour can be interactively warped/modified by dragging the control nodes. The contour widget allows the developer to design a custom vtkPointPlacer to define the placement of points and a custom vtkContourLineInterpolator to provide a way to interpolate between nodes. We have extended these two classes to obtain a contour widget that follows the surface of a 3D object as a real tape measure. When the user interacts with a control node, the measure of the contour is calculated and visualized inside the $3 \mathrm{D}$ scene of $\mathrm{SMA}^{2}$. The customized contour widget has been used also for other feature inside $\mathrm{SMA}^{2}$, such as critical zones definition (Fig. 15) and socket trim-line generation, which are discussed in the following subsections.

$\mathrm{SMA}^{2}$ makes available a third way to get measures of the gap between designed socket and the initial residual limb; in fact, it embeds a software module that permits to visualize the distance between the socket and the residual limb shape using the VTK class named vtkDistancePolyDataFilter. When the user activates this feature, the $3 \mathrm{D}$ point of the socket under the mouse pointer is used through this module to calculate the distance from virtual model of residual limb. The calculated distance is visualized inside $\mathrm{SMA}^{2}$ as shown in Fig. 5. 
Fig. 4 The measures inside red rectangle are relative to perimeters of profiles defined by the three planes

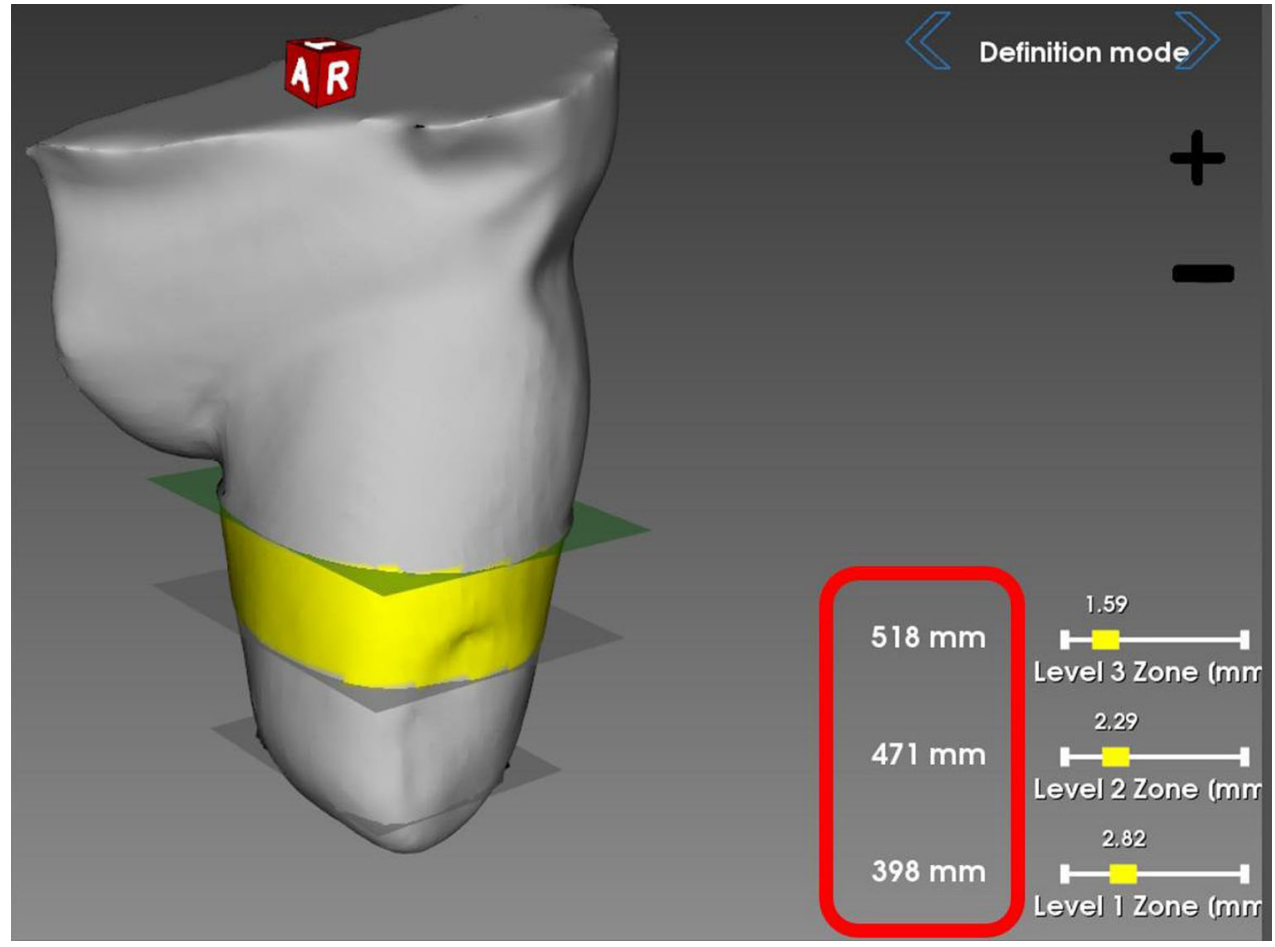

Fig. 5 The distance between the lowest part of the designed socket and the virtual residual limb is shown inside the red rectangle

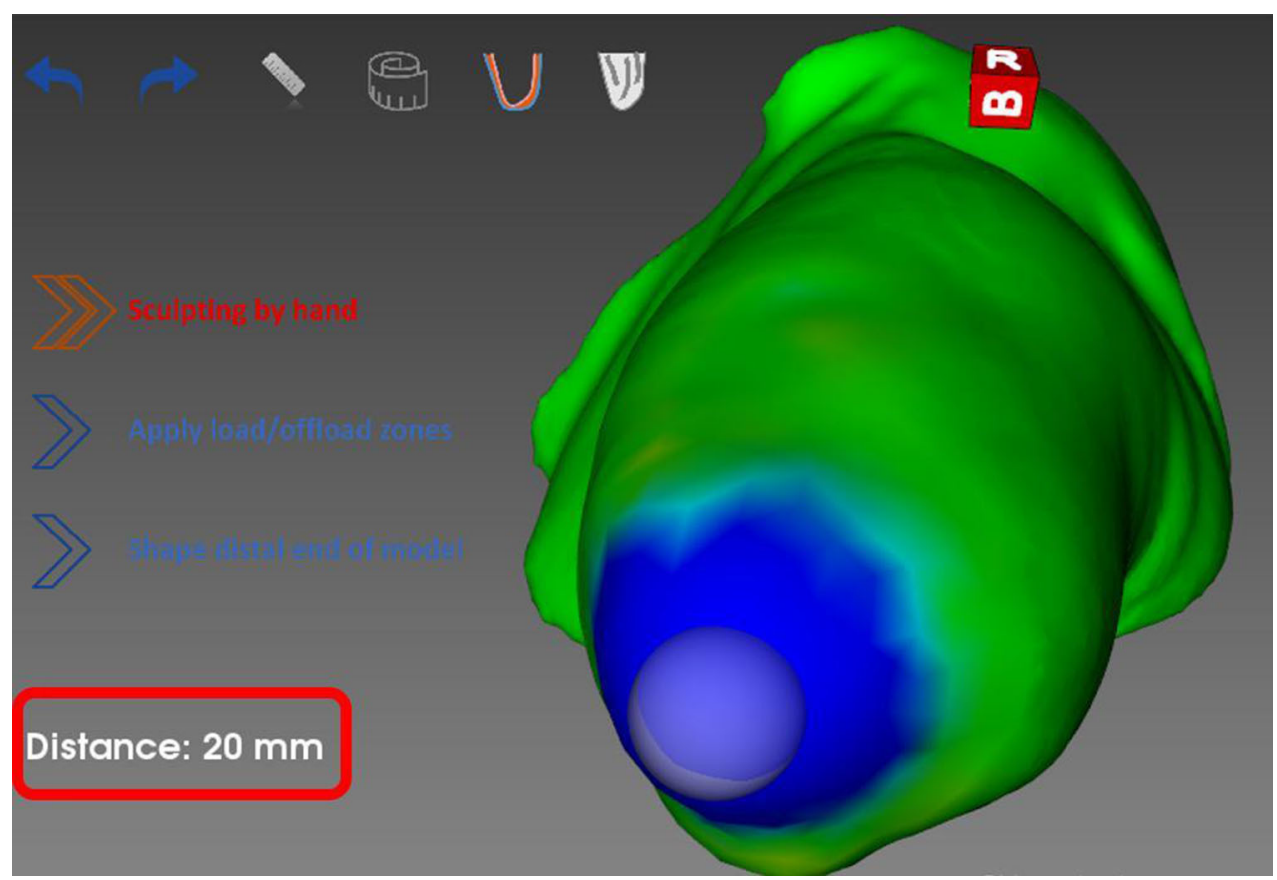

\subsection{Interactive sculpting operations on 3D mesh with self-adaptive topology}

During traditional socket manufacture, main operations are executed by adding or removing chalk from the initial positive model according to measurements, critical zones and patient's data. Therefore, we developed a tool, which emulates sculpting operations on the triangulated meshes. This permitted us to emulate shape deformation through selfadaptive topology algorithms [47] based on local subdivision and decimation of the mesh triangles. Considering classical sculpting tools, such as brush, inflate, smooth, flatten and drag, we developed a set of virtual modelling tools that act on the polygonal mesh in accordance with the tasks executed by the prosthetists. 
Fig. 6 a The initial triangulated structure of a part of the 3D model. Local subdivision (b) and local decimation (c)

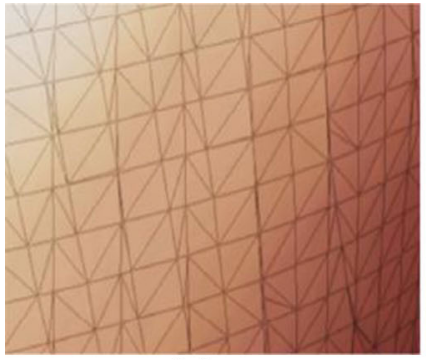

(a)

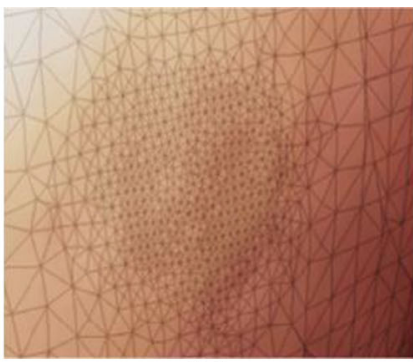

(b)

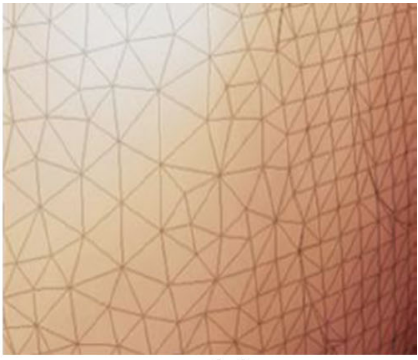

(c)
A class named vtkDynamicSculpt has been developed to permit mesh sculpting through the use of VTK.

\subsubsection{Local subdivision and decimation}

The developed algorithm works directly on each triangle selected during the interaction (Fig. 6a), which is executed by a sphere following the mouse pointer along the $3 \mathrm{D}$ model. According to the sphere radius, vtkDynamicSculpt computes a set of parameters that select edges to be subdivided (Fig. 6b) or decimated (Fig. 6c). After these operations, which are based on dynamic structure inside the mesh topology defined by VTK, the algorithm performs the deformation chosen through the user interface. At present, $\mathrm{SMA}^{2}$ makes available five operations: inflate, deflate, move, flatten and drag. They allow the user to emulate operations as adding/remove material, polishing the surface as really done during the traditional process.

vtkDynamicSculpt has been developed in-house with high modularity to permit to add other modules for executing other types of deformation that could be useful in the future. $\mathrm{SMA}^{2}$ exploits this class in several operations the user can do for socket design. The sculpt tool has been totally developed to execute sculpting operations on the 3D model by hands with no knowledge rules. The deformation tool exploits vtkDynamicSculpt in order to execute modification according to highlighted critical zones. Lower zone definition tool defines lower part on the designed socket by exploiting decimation and subdivision algorithm.

Finally, vtkDynamicSculpt makes available Undo ( ) and Redo () methods, which are exploited inside for each operation performed on the $3 \mathrm{D}$ model in every single virtual tool.

\subsubsection{Deformation algorithms}

As mentioned, $\mathrm{SMA}^{2}$ embeds five different deformations emulating possible operations the technician usually executes during socket design. Inflate and deflate allows adding/ removing virtual material by following the surface. The selected points are translated along their normal according to the intensity of the operation. Once selected points have been translated, a Laplacian smooth filter is applied to reorder the deformed part of the mesh [46] and maintain the 3D triangular mesh as manifold mesh. Flattening and Smoothing are totally based on a set of algorithms already exploited in other application, such as Autodesk MeshMixer [2]. Drag operation allows moving the selected zone by following mouse direction. The algorithm is based on the mathematical model described in [47]. This virtual operation can be useful for adding virtual mesh where the user needs to create shapes without following the existent surface as done by inflate/deflate operations.

\subsection{Automatic trim-line generation}

The socket trim-line is the upper contour of the socket, which requires high skills because it influences the good fitting of the socket. Usually, orthopaedic technicians define a set of templates to easily define the trim-line for both transfemoral and transtibial amputation. Using the above mentioned contour widget, we adopted the same approach and we defined four templates for TT and one template for TF (Figs. 7 and 8 ). By the way, the high modularity of this approach allows easily adding new templates.

Socket trim-line generation is based on patient's data and defined critical zones. SMA ${ }^{2}$ considers, for both transfemoral and transtibial amputations, some zones defined by the user to arrange the path of the contour widget along the modelled 3D model. The trim-line is composed by a curved line with which the user can interact using a set of control points positioned along the path. The user can add/remove/update a node along the trimline by using mouse and keyboard. Once defined the main positions along the 3D model, all nodes are automatically generated by the developed algorithm.

The developed algorithm calculates the positions by starting from two height values, which define the highest (i.e., $h_{\max }$ ) and the lowest (i.e., $h_{\min }$ ) heights of the whole contour. Both values have been defined in the following way:

- For a TT amputation, $\mathrm{h}_{\min }$ is the height of the center of mass of the patella zone and the $h_{\max }$ is calculated as the distance between the lowest point of the residual limb 


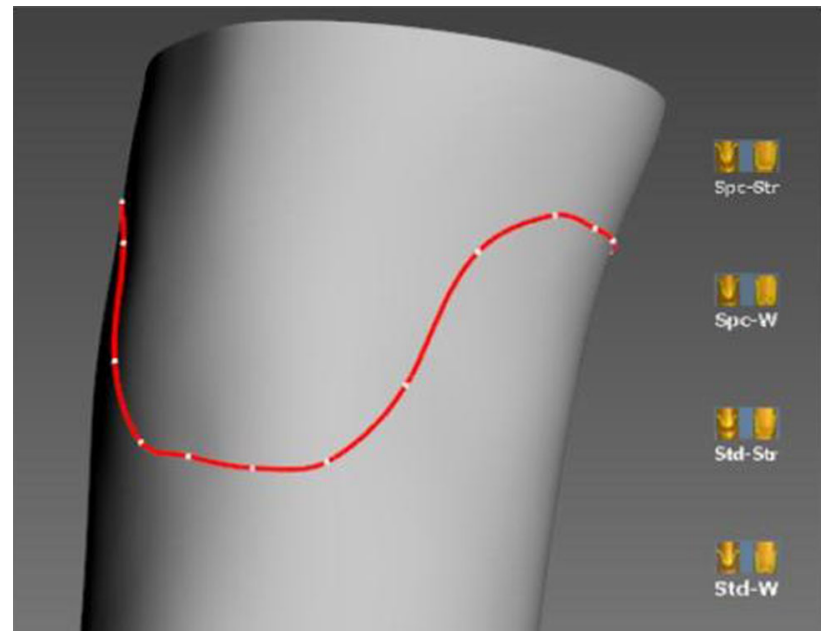

Fig. 7 Trim-line automatically generated by clicking the first template in the right side of the virtual tool
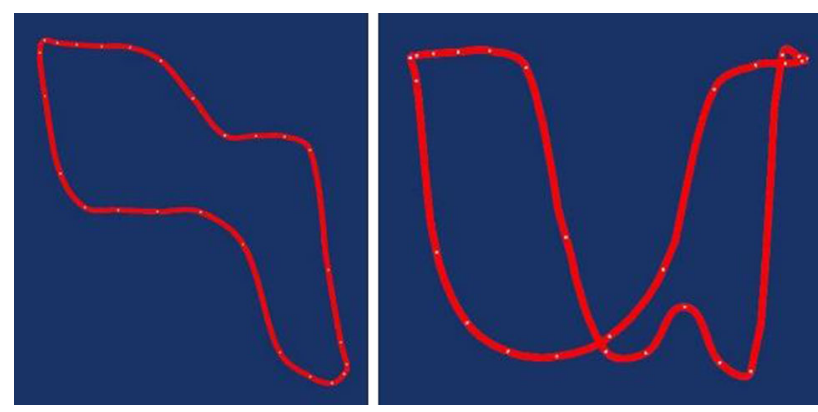

Fig. 8 A template of a trim-line for a socket of a TF amputation and a template for a socket of a TT amputation

and the knee, which is a value defined from the patient's data.

- For a TF amputation, $\mathrm{h}_{\min }$ is the highest point of the inguinal canal off-load zone and $\mathrm{h}_{\max }$ is the highest position defined for the upper edge containment zone.

Then, $\mathrm{h}_{\min }$ is calculated as fraction of $\mathrm{h}_{\max }$. The algorithm uses the values of templates, which have been defined in percentages, to calculate absolute values of heights according to the size of the $3 \mathrm{D}$ model. The template values take a value $\mathrm{u}_{\text {template }}$ between 0 and 24 because the user can create template in very simple way instead of using decimal values between 0 and 1 . The maximum value associated to the template defines $\mathrm{u}_{\mathrm{dim}}$. The number of nodes of all templates values is 20 .

$$
\begin{aligned}
& u \%=\frac{u_{\text {template }}}{u_{\text {dim }}} \\
& h_{\text {node } \%}=h_{\max } \%-u \% *\left(100-h_{\min \%}\right) \\
& h_{\text {node }}=h \max * \frac{h_{\text {node } \%}}{100}
\end{aligned}
$$

The value $u \%$ defines a normalization between 0 and 1 of each $u_{\text {template }}$ value (Eq. (1)). The Eqs. (2) and (3) calculate $\mathrm{h}_{\text {node }}$ for each node. When all absolute heights have been calculated, assuming the $y$-axis as the vertical axis of the 3D model, we initially create a $\mathrm{z}-\mathrm{x}$ planes intersecting the $3 \mathrm{D}$ model in $h_{\max }$. At this height, we create a circumference whose center corresponds to the center of the profile belonging the plane. Each point of circumference is moved toward the center of the circumference itself until the point inter-sects the surface, the intersected point is used to define the position of each node by changing the y value with $h_{\text {node }}$. Finally, the vtkContourWidget repositions the nodes along the surface in automatic way and the trim-line is correctly generated.

\section{The new modelling procedure}

The new modelling procedure is composed by three main steps. The first step is relative to patient's data acquisition that consists on getting anthropometric measurements, lifestyle information and 3D model of amputated lower limb. These data are exploited inside $\mathrm{SMA}^{2}$ to design virtual socket model. Virtual tools of $\mathrm{SMA}^{2}$ carry out several modelling operations. The first is the preliminary modelling to define 3D initial shape of socket model according to patient's data. Then, a customized modelling procedure must be done for assessments of the final socket shape (e.g., manual shaping). Finally, the socket geometry is completed with standard details (e.g., thickness of socket, trim-line of the upper part of the socket and position of valve) as well as a finite element simulation is done for evaluating behaviours between designed socket and residual lower limb and, if it needs, further improving the socket. Whole modelling procedure is deeply described in the following subsections.

\subsection{Acquisition of patient's data}

Initially, the orthopaedic technician starts inserting patient data (e.g., weight, muscles, tonicity, skin conditions and residual limb stability), which are necessary to apply design rules and/or suggests the most appropriate procedure to the user during each step of the virtual socket design (Fig. 9).

After inserting patient's data, $\mathrm{SMA}^{2}$ requires the 3D model of the residual limb. Two options are available: geometric model generated from MRI images or with a 3D scanner.

In the first case, two models are automatically generated representing respectively external shape of residual limb and the internal bones (Fig. 10). We have developed a software module which automatically reconstruct 3D models of both skin and bones by starting from MRI volume. The software algorithm can extract an ordered 3D point cloud by MRI images which are processed to determinate contours of inter- 
Fig. 9 Patients history

Fig. 10 Automatic 3D reconstruction from MRI volume of an above amputated lower limb
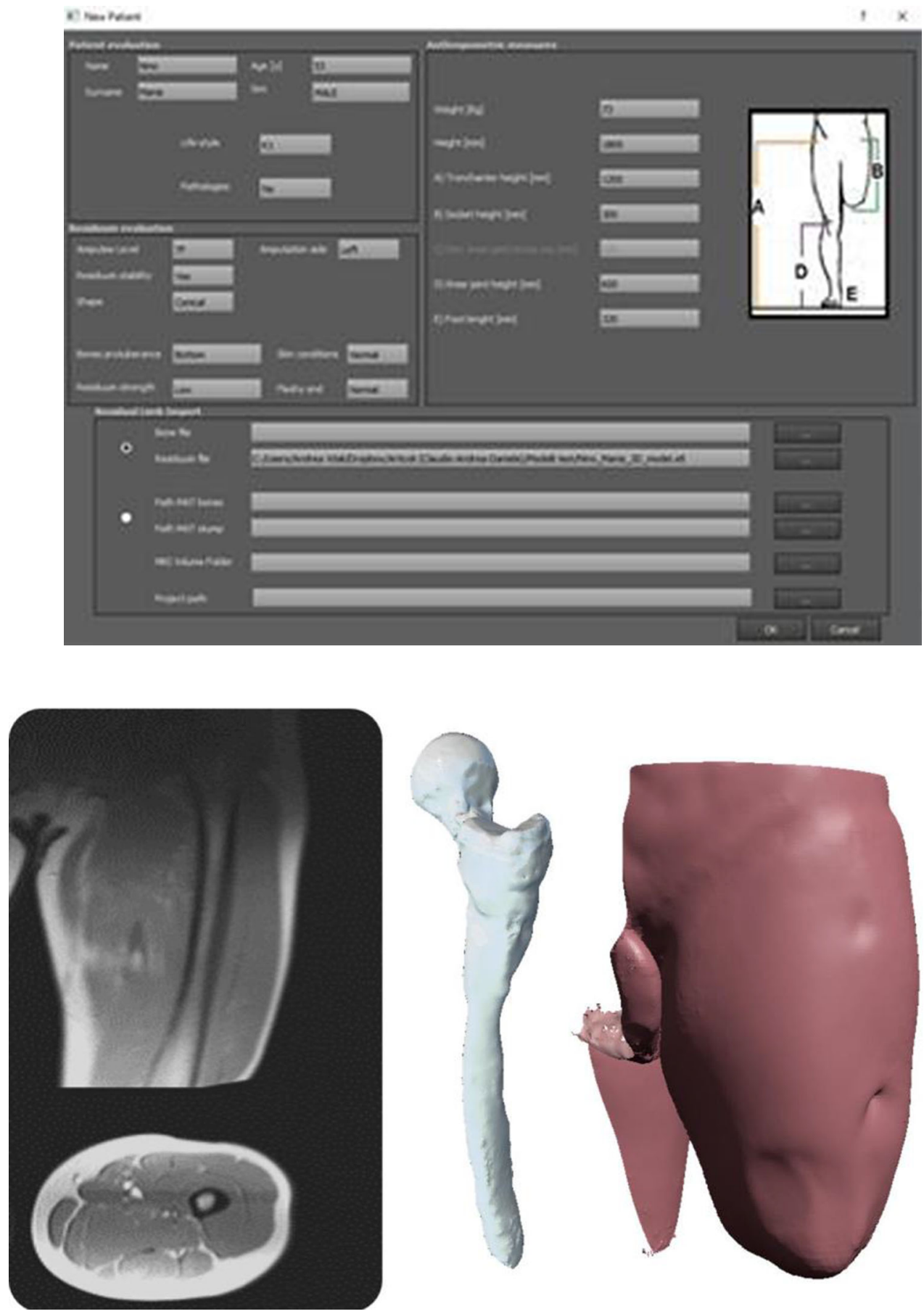

ested tissues. The ordered points cloud is used to create a NURBS surface by fitting algorithm using a software library developed by us to manage NURBS surface through VTK (i.e., SimplyNURBS) [9,12]. This approach allows getting 3D models in two different file formats: STL file format for modelling with $\mathrm{SMA}^{2}$ and IGES file format for finite element simulation.

In the latter, the external surface of the residual limb is acquired with Microsoft Kinect v1 and corresponding tri- angulated model is generated with Skanect (Fig. 11) [45]. During the 3D acquisition, the patient is in an upright position to acquire the 3D model of the residual limb without deformations of muscles, which can occur when the patient is sitting or in another position. Therefore, another person moves the Kinect around the residual limb to the whole human district. 

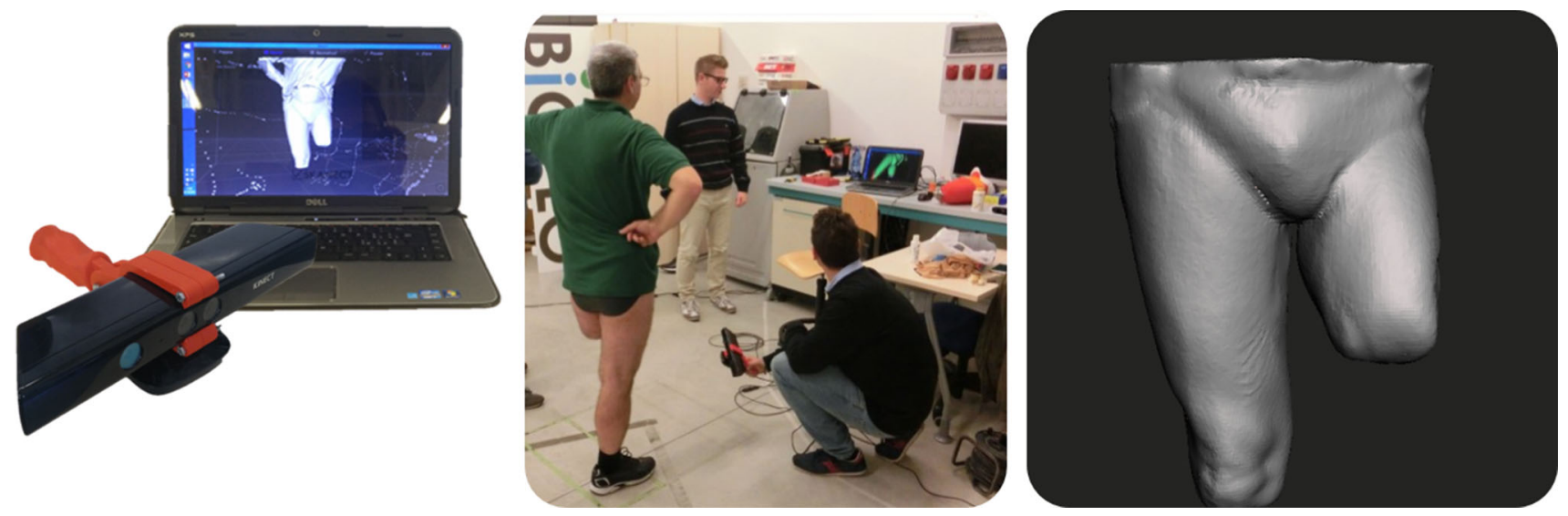

Fig. 11 3D scanning procedure using low cost IT solution

\subsection{Modelling}

As mentioned in the previous sections, a set of virtual modelling tools have been implemented and, according to their final purpose, subdivided into three groups: preliminary modelling, customized modelling and completing the socket model.

\subsubsection{Preliminary modelling}

The main operations during preliminary modelling are carried out almost completely in automatic way according to patient characteristics and traditional process. Four modelling tools are available as follows:

- Scaling tool permits to scale the initial model. In fact, in the traditional process the first operation applied on the positive cast is the rasping procedure to reduce the volume. This is done since the socket, manufactured directly on the positive model, must be perfectly close fitting on the patient's residual limb. The technician first identifies on the plaster cast the same reference circumferences previously measured on the patient's residual limb, and then starts to file harmoniously the plaster until these circumferences are reduced of the desired percentages. Through a set of cross section planes are defined as shown in Fig. 3 the user can decide the reduction percentage in correspondence of each of them. The range of percentage varies from 1 to $6 \%$. It is not uniform on the stump, but it starts with $1 \%$ at $40 \mathrm{~mm}$ over the stump top, and it increases gradually going up until the stump upper part. For this procedure the system first identifies the socket top, calculating the lowest point of the geometric model. Then, starting from this point, the system selects 4 reference cross-section planes at a distance of $4 \mathrm{~cm}$ from each other. For each of the used sections it is calculated the middle point and then the distance of each circumfer- ence point is scaled by the appropriate percentage. All the other sections situated between these 4 reference ones are scaled by interpolated values, in relation to their position on the model (Fig. 12).

- Lower part tool that permits to create the lower part of the socket starting from the initial shape of the residual limb. This operation is very important because it defines the lower part of the socket that will be merged with the $3 \mathrm{D}$ model of the socket plug. Also in this case, the distance between the lowest part of the socket and the residual limb can be obtained automatically starting from patient data. Some sliders are available to change the distance and the roundness of the final part of shape without following the automatic procedure (Fig. 13).

- Marker tool allows the user to mark on the surface of the virtual residual limb off-load and load zones with different colours. Figure 14 shows an example of coloured critical zones for a transtibial residual limb. The coloured zones are available every time the orthopaedic technician wants to know what happen to residual limb due to a modification of socket shape.

- Deformation tool emulates the operation of adding/ removing chalk during traditional process and is automatically executed. Starting from highlighted zones, if the zone is an off-load zone the mesh of the marked area is pushed inside of a certain quantity according to patient's characteristics, specifically the residuum tonicity; otherwise the mesh inside the contour is pushed outside. This tool permits also to interactively define and modify the contour line of the load and off-load zones (Fig. 15).

\subsubsection{Customized modelling}

Customized modelling permits to customize and refine the model obtained in the previous phase. The user can proceed with an interactive shape manipulation using the modelling 
Fig. 12 The scaling tool

Fig. 13 Translating the part of the $3 \mathrm{D}$ model under the green plane to prepare an initial zone to define the lower part of final socket

Fig. 14 Critical zones

highlighted with the marker tool
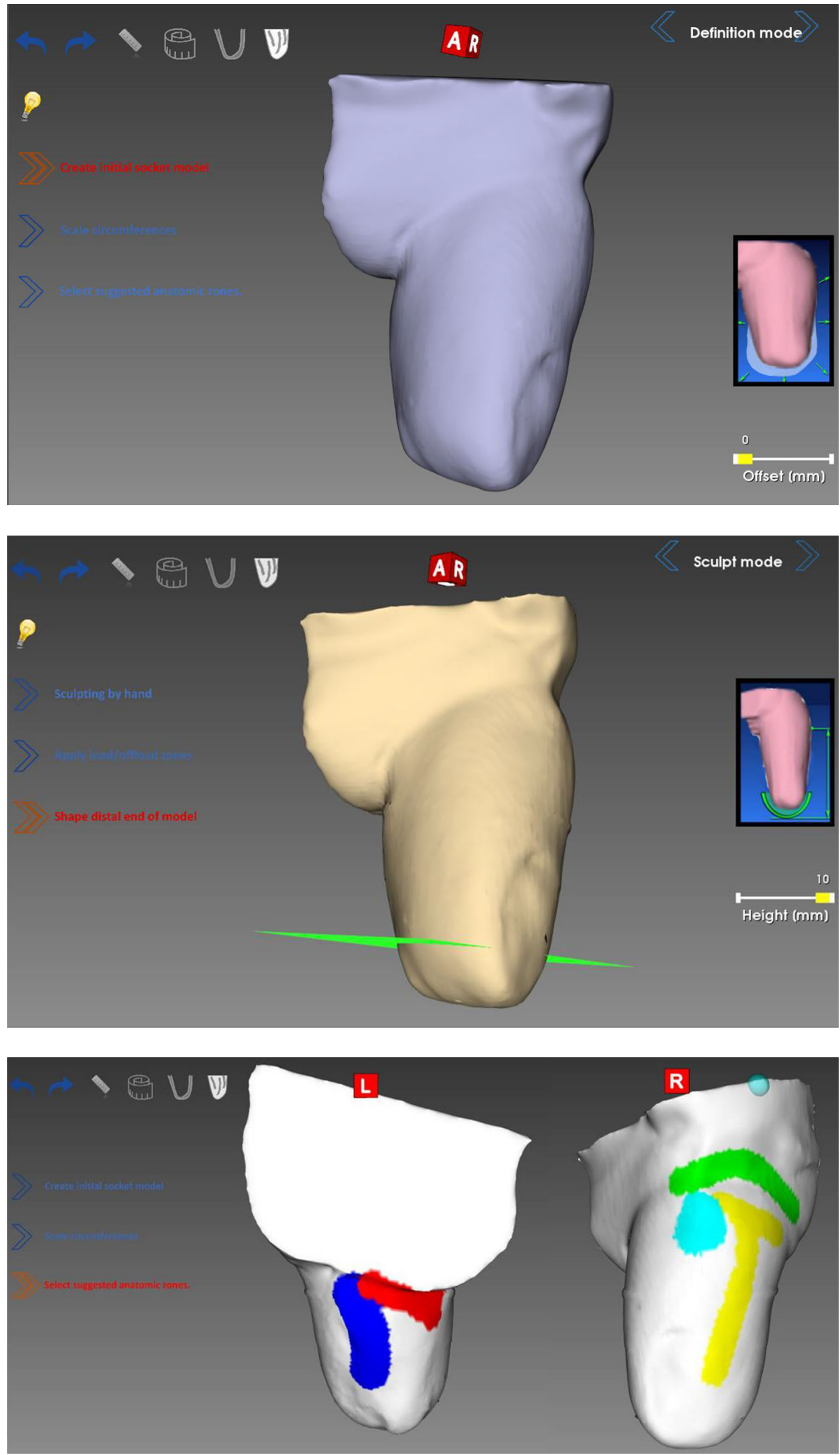
Fig. 15 Some critical zones has been deformed by starting from coloured zones

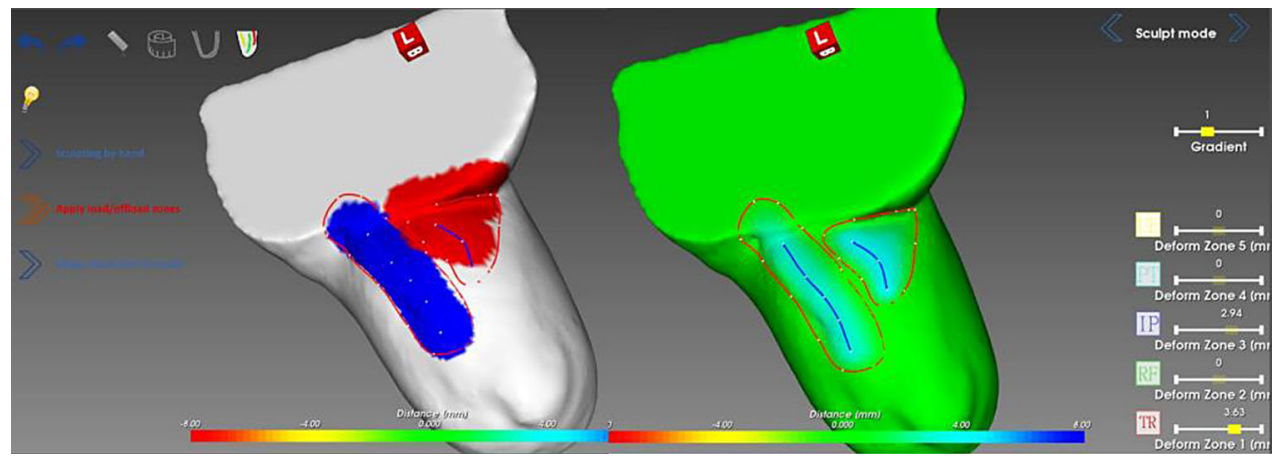

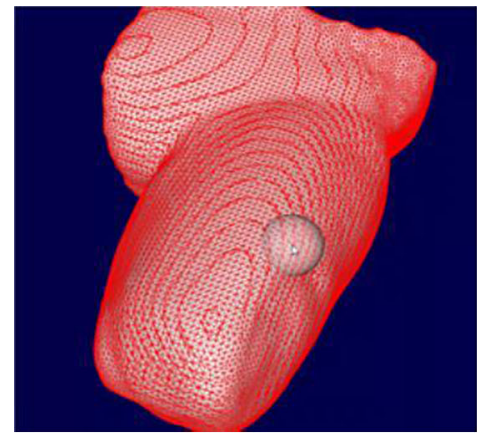

(a)

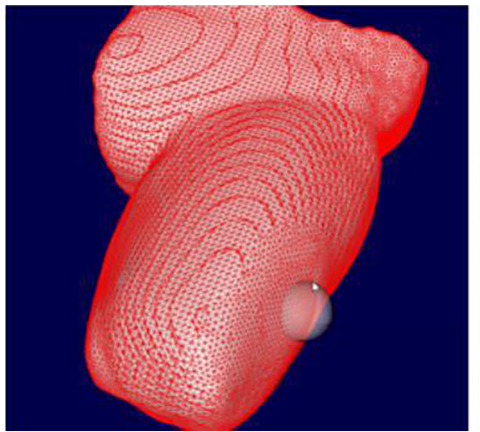

(b)

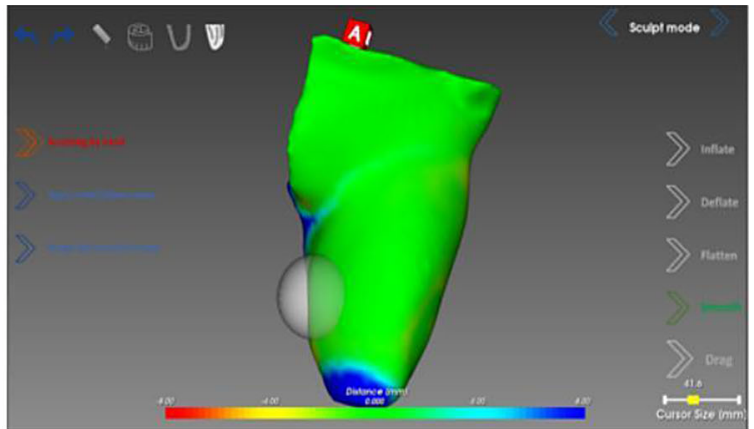

(c)

Fig. 16 a The initial triangulated structure of a part of the 3D model. Local subdivision (b) and local decimation (c)

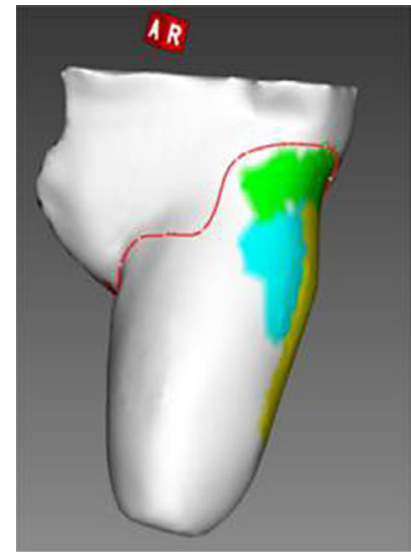

(a)

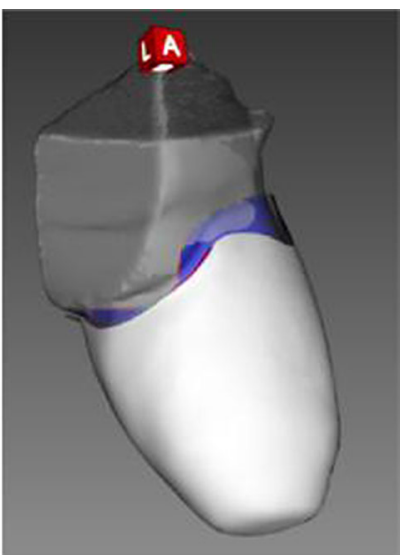

(b)
Fig. 17 Sketch of trim-line and automatic generation of thickness

tool, named sculpt tool. The operations allowed on the mesh are in/deflate, smoothing, flattening and dragging (Fig. 16c). They allow the locally mesh editing to remove details from 3D mesh of residual limb as shown in Fig. 16a, b in which the $3 \mathrm{D}$ mesh is locally smoothed to remove a scar. Also in this case, load and off-loads zones are considered to inform the orthopaedic technicians about the consequences of $3 \mathrm{D}$ modification on the residual limb.

\subsubsection{Completing the socket geometric model}

Completing the socket geometric model consists in shaping the upper edge of the socket, assign a thickness and create the hole to assemble the valve.

The first operation can be executed in an automatic or semi-automatic way. The system provides different templates for the socket trim-line identified in collaboration with orthopaedic technicians previously mentioned. The user selects the template and the trim-line contour is automatically generated on the mesh of socket. Then, the user can modify the trim-line moving the control points along the surface of the 3D residual limb model. Once the trim-line has been defined (Fig. 17a), SMA $^{2}$ removes the upper part of the model and the final shape of socket is created (Fig. 17b). At this point, the operation of socket thickening is automatically executed by the system creating an offset outward. This offset represents the socket external surface and the offset distance is the final socket thickness. The final socket thickness is usually uniform and calculated using the following empirical formula:

$\mathrm{ST}[\mathrm{mm}]=\mathrm{PW}[\mathrm{kg}] / 20$

where, $\mathrm{ST}$ is the socket thickness, PW is the patients weight.

Finally, the last tool permits to create the hole to assemble the valve. To add the hole for the valve, 3D modelling Boolean operators have been exploited. The difference oper- 

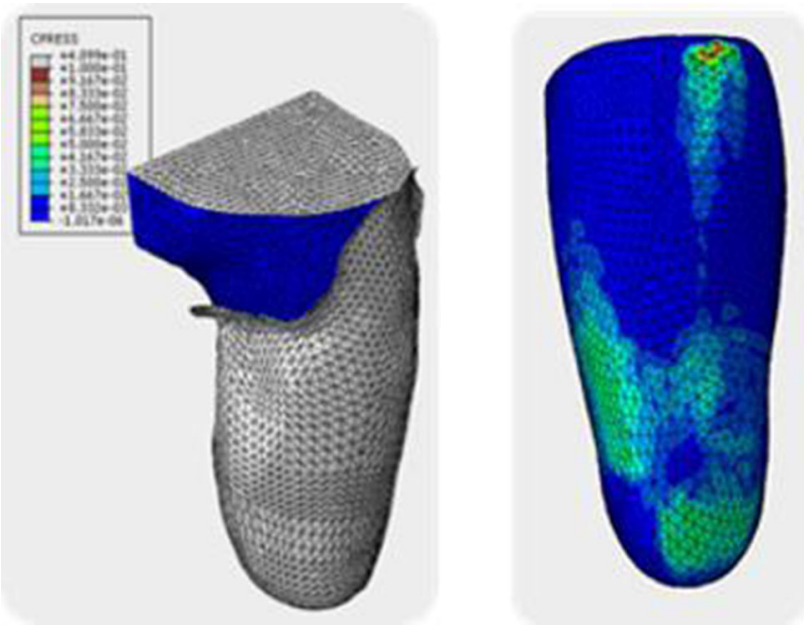

Fig. 18 Finite element analysis

ator has been applied between the socket mesh and a 3D cylinder with the same diameter of the chosen valve. The cylinder is positioned along the surface of the designed socket according to position chosen by mouse pointer. When the user has defined the correct position, he/she clicks the mouse and the difference operator is applied and a hole is added to the socket. Also in this case, the virtual tool has been developed using VTK.

Once the first 3D socket model is generated, the system automatically executes the FE analysis to verify contact pressure and then optimize the socket shape (Fig. 18). FEA results, imported in SMA, are analysed and socket geometry is modified, using the sculpt tool until the optimal shape is reached. Details about FE analysis can be found in [8].

Finally, the socket model can be exported for additive manufacturing. The option for multi-material 3D printing is available and permits to create a socket using different materials according to patient's data and load and off-load zones.

\subsection{Additive manufacturing}

The socket is created with the 3D printer Leonardo 300 Cube" by Meccatronicore. This low-cost 3D printer has two nozzles which can be used to extrude two different materials, e.g. with different stiffness. From a manufacturing point of view handling two materials is not new and is done, for instance, to create support parts which can be easily removed from the product. The challenge is to design where the behaviour of the socket benefits from a harder or softer material. The design process based on $\mathrm{SMA}^{2}$ embeds information about critical zones on the residual limb and measured contact pres-sures [8]. Thus, the structure of the socket can be optimized by matching patient's morphology and applied loads while following medical guidelines. Moreover, additive manufac-turing opens the way to different infill ratio and geometry, so that we can vary product density and mechanical response to loads.

For each critical zone, a contour is automatically created around the zone, then the data relative to contour are used as implicit function in the VTK filter named vtkSelectPolyData, which permits clipping the part of 3D model inside the contour. So, clipped zones are exported in an STL le and the rest of the socket in another one. These two files are both loaded inside the 3D printer software to associate each to a different material.

$\mathrm{SMA}^{2}$ includes a module able to export two different STL files (Fig. 19a,b), e.g. one for the hard zones of the socket and the other one for soft zones. Both files are used by 3D printer software application to allow multi-material printing (Fig. 19c). In this way, the socket results more suitable for the anatomical behaviour of the residual limb.

\section{Case study}

The patient is a male with an above knee amputation and he is 53 years old. The first step has been to fill the form relative to anthropometric data and his lifestyle. The form is also used to compile the digital form of $\mathrm{SMA}^{2}$, which permits to design the socket starting from the 3D model of patient's residual limb. Several sections compose it; each of them contains information useful for a particular step of the design procedure. As depicted in Fig. 10, the modules relative to the definition of lower part of the 3D model will be used by $\mathrm{SMA}^{2}$ for socket design as well as the final assembly of the whole lower limb prosthesis. The circumferences along the thigh of the residual limb and the anthropometric data are exploited into $\mathrm{SMA}^{2}$ to permit the automatic and semiautomatic operations for each virtual tool. The other data are used during acquisition of the patients gait.

Both acquisition and reconstruction of the residual limb from MRI images were already available [9]; therefore, a solution has been tested based on 3D scanning acquisition using Microsoft Kinect v1 and the Skanect application. The residual limb has been acquired by keeping the muscle of the residual limb in relaxed condition. This is very important because the shape of the residual limb can suffer from a big deformation that makes impossible the socket design with the 3D virtual model (Fig. 20a). Scanned 3D model of the residual limb has been exported by Skanect in STL file format. The STL file will be used as initial 3D triangular mesh model for starting the socket design (Fig. 20b).

The triangular mesh of the residual limb is loaded inside SMA $^{2}$ and the scaling tool scales the initial 3D model starting from the measurements obtained by the form previously introduced. Then, the marker tool allows the identification of load and off-load zones and thus, the deformation tool has been used to deform marked zones (Fig. 15). 
Fig. 19 a The shell structure to be printed with hard material and $\mathbf{b}$ the critical zones with soft material. 3D printer software loads both STL files together (c)

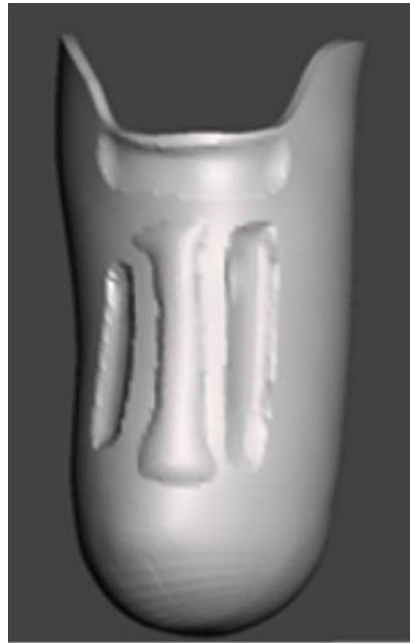

(a)

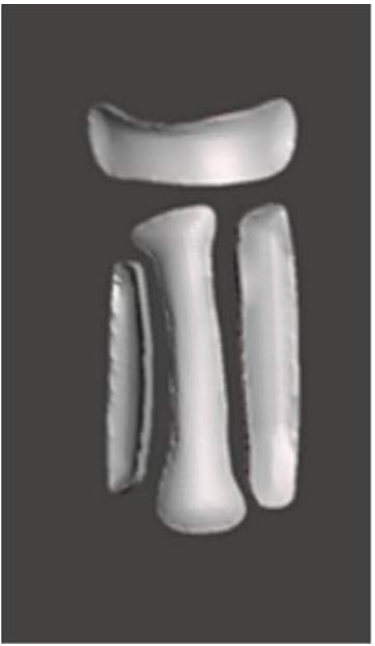

(b)

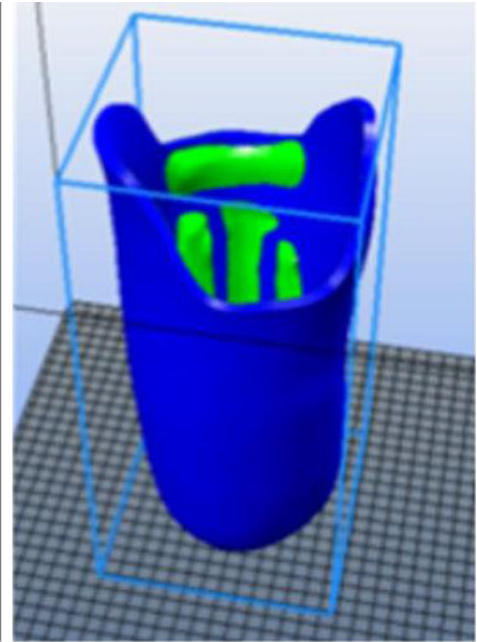

(c)

Fig. 20 3D acquisition using Microsoft Kinect and Skanect without support under the residual limb

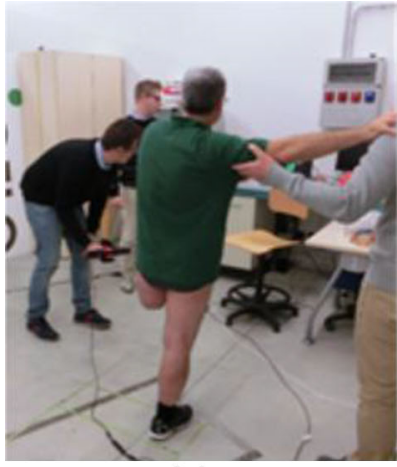

(a)

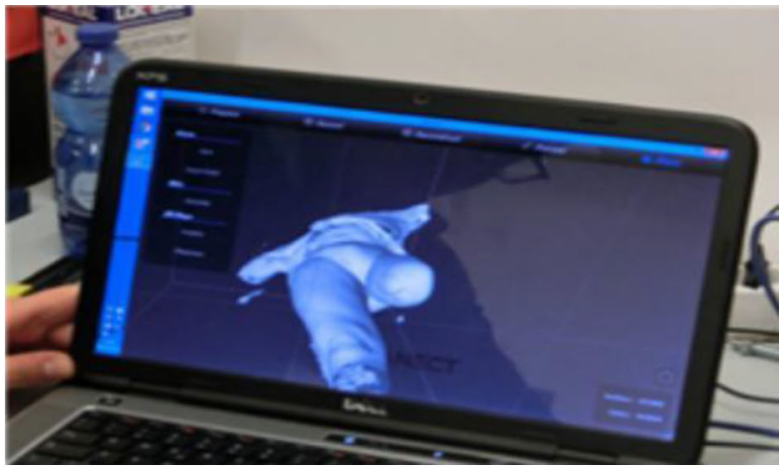

(b)
The lower part of the socket is generated (Fig. 13) and mesh modelling is done to remove some useless details of the initial 3D triangular mesh using the sculpt tool (Fig. 16c). The trim-line of upper part of the socket is automatically created (Fig. 21a) and the thickness applied (Fig. 21b). Finally, the, the plug is merged with the 3D socket model, the hole for the valve added and the final STL file of the 3D socket generated (Fig. 21c). The STL file is used in the next step in order to permit a single material 3D printing (Fig. 21d).

The socket has been realized with the FDM printer (i.e., Meccatronicore Leonardo) and, then assembled with the valve (Fig. 21d) and connectors to the whole prosthesis. The patient wore it and patients feedback was good enough to complete the donning of the socket (Fig. 22).

Once assembled the complete prosthesis, the patient worn it and walked along a straight line. He reported that there was a tight-fitting zone in the ischial area and this caused pain after few steps. Even if the designed socket was not good for a normal gait and modifications are required, the patient commented that the $3 \mathrm{D}$ printed socket can potentially substitute the thermoformed cemplex traditionally realized at the orthopedic lab.

\section{Conclusions}

The performance of a prosthesis for lower limb amputation heavily depends on the experience and skills of the prosthetist. Most of its component are standard, such as the knee or the foot, and can be selected from commercial catalogues. Instead, the socket, and sometimes also the liner, is the customized component and totally hand made. It is the most critical component and is the interface with the human body. The final comfort and function of the whole prosthesis mostly depends on its quality. Available commercial prosthetic tools can support some specific steps of the process, but they still rely on a traditional design paradigm and do not offer any kind of assistance or suggestions to the user, and the technician knowledge and experience are still required. This research work presents $\mathrm{SMA}^{2}$ an innovative prosthetic CAD system specifically conceived to design the socket. It has been designed and implemented following a low-cost philosophy and open source libraries to provide a computeraided environment affordable also by small orthopaedic labs Two procedures have been developed to reconstruct the 3D models of the residual limb around which the socket model 


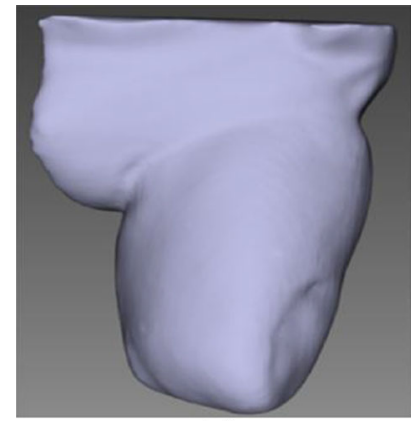

(a)

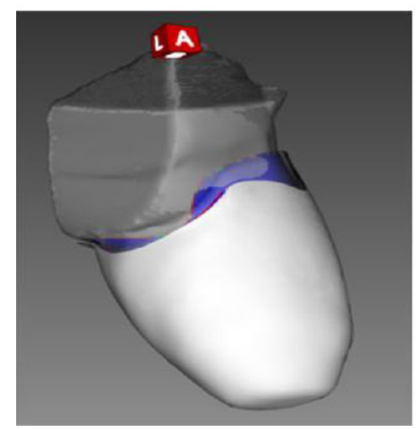

(c)

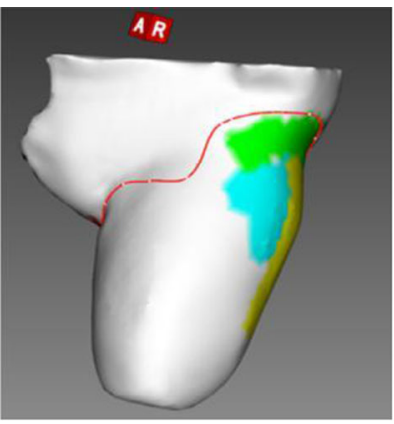

(b)

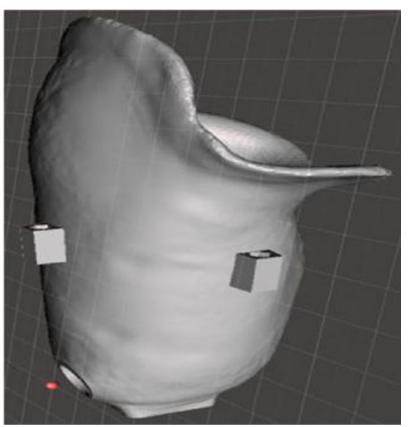

(d)
Fig. 21 Some steps of the innovative procedure using $\mathrm{SMA}^{2}$ during the case study

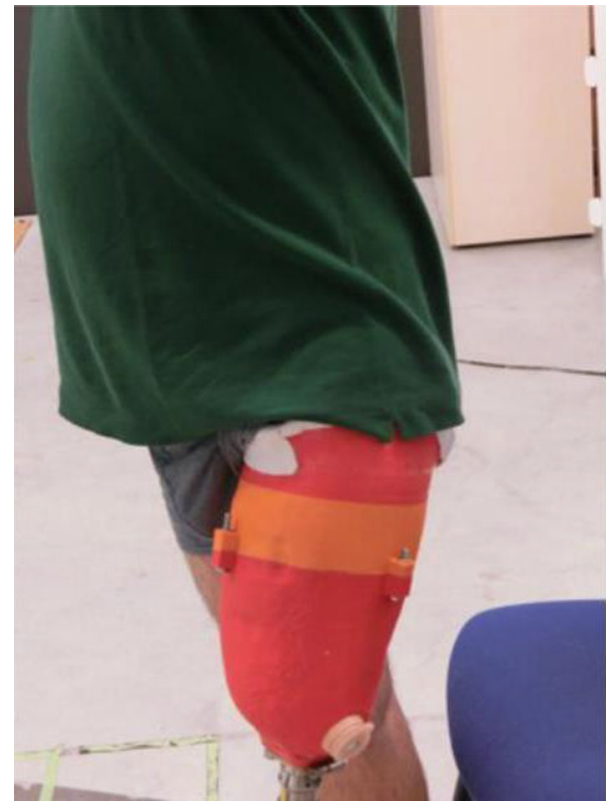

Fig. 22 Donning of the 3D printed socket

is designed. The first one is based on a low-cost scanner 3D, i.e. the Microsoft Kinect; the second one permits to automatically reconstruct the virtual model from MRI images. Starting from the virtual model of the residual limb, SMA ${ }^{2}$ makes available a set of virtual modelling tools that emulate the operations performed by the technicians during the tradi- tional hand-made manufacturing process. A new interaction style has been implemented to allow the user to interact by hands using hand-tracking and haptic devices. A data driven multi-material 3D printing approach has been developed to realize the physical socket using the FDM technology. The whole digital platform has been tested with a transfemoral amputee. Once modelled with $\mathrm{SMA}^{2}$, the socket has been realized using the FDM technology. The patient wore the physical prototype that has been considered comfortable. The technicians appreciated the whole system and the possibility to use the framework to simplify the work of experienced technicians and train junior designers who can learn more quickly about lower limb prosthesis design.

To conclude, interactive design and manufacturing approach permitted us to realize a computer-aided platform by which the prosthetist can model the socket shape using ad hoc developed modelling tools (e.g., sculpt tool) interacting with the residual geometric model and manufacture it by using additive manufacturing. This makes the design process more reliable and repeatable and at the same time still enough like the traditional process to be accepted by the prosthetists. The same approach can be easily extended to product highly customized, such as clothing.

\section{References}

1. 3DSlicer. Open source software plat-form for medical image informatics. https://www.slicer.org/

2. Autodesk. Meshmixer. http://www.meshmixer.com/

3. Barone, S., Paoli, A., Razionale, A., Savig-nano, R.: Computer aided modelling to simulate the biomechanical behaviour of customised orthodontic removable appliances. Int. J. Interact. Des. Manuf. 10(4), 387-400 (2016)

4. Braganca, S., Arezes, P., Carvalho, M.: An overview of the current three-dimensional body scanners for anthropometric data collection. Occup Saf Hyg III, 149-153 (2015)

5. Brandt, M.: Laser Additive Manufacturing: Materials, Design, Technologies, and Applications. Wood-head Publishing, Cambridge (2016)

6. Calvo, I., Lopez, F., Zulueta, E., Gonzalez-Nalda, P.: Towards a methodology to build virtual reality manufacturing systems based on free open software technologies. Int. J. Interact. Des. Manuf. 11(3), 569-580 (2016)

7. Chen, X., Xu, L., Wang, H., Wang, F., Wang, Q., Kikinis, R.: Development of a surgical navigation system based on $3 \mathrm{~d}$ slicer for intraoperative implant placement surgery. Med. Eng. Phys. 41, 81-89 (2017)

8. Colombo, G., Comotti, C., Redaelli, D. F., Regaz-zoni, D., Rizzi, C., Vitali, A.: A method to improve prosthesis leg design based on pressure analysis at the socket-residual limb interface. In: Volume 1A: 36th Computers and Information in Engineering Conference (2016)

9. Colombo, G., Facoetti, G., Rizzi, C., Vitali, A., Zanello, A.: Automatic $3 \mathrm{~d}$ reconstruction of transfemoral residual limb from MRI images. In: Lecture Notes in Computer Science, pp. 324-332 (2013) 
10. Colombo, G., Facoetti, G., Morotti, R., Rizzi, C.: Physically based modelling and simulation to innovate socket design. Comput. Aided Des. Appl. 8(4), 617-631 (2011)

11. Colombo, G., Facoetti, G., Rizzi, C.: A digital patient for computeraided prosthesis design. Interface Focus 3(2), 20120082-20120082 (2013)

12. Colombo, G., Facoetti, G., Rizzi, C., Vi-tali, A.: SimplyNURBS: a software library to model nurbs for medical applications. Comput. Aided Des. Appl. 12(6), 794-802 (2015)

13. Comotti, C., Regazzoni, D., Rizzi, C., Vitali, A.: Multi-material design and 3D printing method of lower limb prosthetic sockets. In: Proceedings of the 3rd 2015 Workshop on ICTs for improving Patients Rehabilitation Research Techniques-REHAB '15 (2015)

14. Domnguez, M.G., Hernandez, C., Ruisoto, P., Juanes, J.A., Prats, A., Hernandez, T.: Morpho-logical and volumetric assessment of cerebral ventricular system with $3 \mathrm{~d}$ slicer software. J. Med. Syst. 40(6), 154 (2016)

15. Fantini, M., De Crescenzio, F., Ciocca, L.: De-sign and rapid manufacturing of anatomical pros-thesis for facial rehabilitation. Int. J. Interact. Des. Manuf. 7(1), 51-62 (2012)

16. Fasel, J.H.D., Aguiar, D., Kiss-Bodolay, D., Montet, X., Kalangos, A., Stimec, B.V., Ratib, O.: Adapting anatomy teaching to surgical trends: a combination of classical dissection, medical imaging, and 3d-printing technologies. Surg. Radiol. Anat. 38(3), 361-367 (2016)

17. Fok, W.W.: Opening up the future of open source: from open innovation to the internet of things for the built environment. Archit. Des. 86(5), 116-125 (2016)

18. Ganry, L., Hersant, B., Quilichini, J., Leyder, P., Meningaud, P.: Use of the 3D surgical modelling technique with open-source software for mandibular fibula free flap reconstruction and its surgical guides. J. Stomatol. Oral Maxillofac. Surg. 118(3), 197-202 (2017)

19. Gao, Y., Duan, H.: A survey of the virtual re-building of manufacturing process based on virtual and reality technologies. In: Anti-counterfeiting, Security, and Identi cation (2012)

20. Gao, Y.W., Zhang, Y., Ramanujan, D., Ramani, K., Chen, Williams, C.B., Wang, C.C.L., Shin, Y.C., Zhang, S., Zavattieri, P.D.: The status, challenges, and future of additive manufacturing in engineering. Comput. Aided Des. Appl. 69, 65-89 (2015)

21. Goldman, L.W.: Principles of CT and CT technology. J. Nucl. Med. Technol. 35(3), 115-128 (2007)

22. Haak, D., Page, C.-E., Deserno, T.M.: A sur-vey of DICOM viewer software to integrate clinical research and medical imaging. J. Digit. Imaging 29(2), 206-215 (2016)

23. Hebert, K.V., Keen, R.S., King, D.R., Shady, S.F.: Gait-Monitoring wearable technology for transtibial prosthetics. In Volume 3: Biomedical and Biotechnology Engineering, ASME 2016 International Mechanical Engineering Congress and Exposition (2016)

24. Héno, R., Chandelier L.: 3D digitization by laser scanner. In 3D Modeling of Buildings, John Wiley \& Sons, Inc., Hoboken, NJ, USA, pp. 85-124 (2014)

25. InVesalius. Invesalius software application. https://www.cti.gov. br/en/invesalius

26. Karbowski, K., Szczybura, M., Sujka, W.: 3D scanner for medical applications. Mechanika 12, 1904-1905 (2016)

27. Lanzotti, A., Grasso, M., Staiano, G., Mar-torelli, M.: The impact of process parameters on me-chanical properties of parts fabricated in pla with an open-source 3-d printer. Rapid Prototyp. J. 21(5), 604-617 (2015)

28. Lipton, M.L.: Totally Accessible MRI: A User's Guide to Principles, Technology, and Applications. Springer, Berlin (2010)

29. Materialise. Services for medical and manufacturing. http://www. materialise.com/en/home

30. MITK. The medical imaging interaction toolkit (mitk)—mitk.org. http://mitk.org/wiki/MITK
31. Mooney, J.J., Sarwani, N., Coleman, M.L., Fotos, J.S.: Evaluation of three-dimensional printed materials for simulation by computed tomography and ultrasound imaging. Simul. Healthc. 12(3), 182188 (2017)

32. Narizzano, M., Arnulfo, G., Ricci, S., Toselli, B., Tisdall, M., Canessa, A., Fato, M.M., Cardi-nale, F.: Seeg assistant: a 3dslice extension to support epilepsy surgery. BMC Bioinform. 18(1), 124 (2017)

33. Niatech. Niatech application. http://niatech.org/

34. OpenCascade. CAD/CAM Softeware Development Kit. https:// www.opencascade.com/

35. Openhub.net. The devide open source project on open hub. https:// www.openhub.net/p/DeVIDE

36. Patalano, S., Lanzotti, A., Del Giudice, D., Vi-tolo, F., Gerbino, S.: On the usability assessment of the graphical user interface related to a digital pattern software tool. Int. J. Interact. Des. Manuf. 11(3), 457-469 (2017)

37. Qt. Cross-platform software development for embedded \& desktop. https://www.qt.io

38. RadiAnt. Radiant DICOM viewer. https://www.radiantviewer. $\mathrm{com} /$

39. Rodin4D. Cad/cam solution for prosthetics and orthotics. http:// rodin 4 d.com/en

40. Russell, J., Cohn, R.: Osirix. Book on Demand Limited, (July 2012)

41. Sanchez-Gomez, S., Herrero-Salado, T.F., Maza-Solano, J.M., Ropero-Romero, F., Gonzalez-Garc a, J., Ambrosiani-Fernandez, $\mathrm{J}$.: Improved planning of endoscopic sinonasal surgery from 3dimensional images with osirix $\mathrm{R}$ and stereolithog-raphy. Acta Otorrinolaringol. (English Edition) 66(6), 317-325 (2015)

42. Sengeh, D.M., Herr, H.: A Variable-Impedance prosthetic socket for a transtibial amputee designed from magnetic resonance imaging data. J. Prosthet. Orthot. 25(3), 129-137 (2013)

43. Sengeh, D.M., Moerman, K.M., Petron, A., Herr, : Multi-material 3 -d viscoelastic model of a transtibial residuum from in-vivo indentation and mri data. J. Mech. Behav. Biomed. Mater. 59, 379-392 (2016)

44. Siavashpour, Z., Aghamiri, M.R., Jaberi, R., Dehghan-Manshadi, H.R., Sedaghat, M., Kirisits, C.: Evaluating the utility of $3 \mathrm{~d}$ slicer" as a fast and independent tool to assess intrafractional organ dose variations in gynecological brachytherapy. Brachytherapy 15(4), 514-523 (2016)

45. Skanect. Skanect 3d scanning software by occipital. http://skanect. occipital.com/

46. Sorkine, C. O., Cohen-Or, D., Lipman, Y., Alexa, M., Rossl, Seidel, H.-P.: Laplacian surface editing. In Proceedings of the 2004 Eurographics/ACM SIGGRAPH Symposium on Geometry Processing-SGP '04 (2004)

47. Stanculescu, L., Chaine, R., Cani, M.-P.: Freestyle: sculpting meshes with self-adaptive topology. Comput. Graph. 35(3), 614 $622(2011)$

48. Themo Fisher Scientific. Amira for Preclinical Imaging. https:// www.fei.com/software/amira-for-preclinical-imaging/

49. Vorum. Cad cam for prosthetics and orthotics-vorum. http:// vorum.com/

50. VTK. The visualization tool kit. https://www.vtk.org/

51. Wang, C.C.L.: Geometric Modeling and Reasoning of HumanCentered Freeform Products. Springer, Berlin (2013)

52. WilloWood. Willowood product and serivices. https://www. willowwoodco.com/

53. Wimpenny, D.I., Pandey, P.M., Ku-mar, L.Jyothish: Advances in 3D Printing \& Additive Manufacturing Technologies. Springer, Singapore (2016) 